\title{
IMPACT FORCE IDENTIFICATION USING THE MODAL TRANSFORMATION METHOD IN COLLOCATED AND NON- COLLOCATED CASES
}

\author{
Abdul Ghaffar Abdul Rahman ${ }^{1}$, Khoo Shin Yee $^{2^{*}}$, Zubaidah Ismail ${ }^{3,4}$, Kong Keen \\ Kuan $^{2}$, Ong Zhi Chao ${ }^{2,4}$,Chong Wen Tong ${ }^{2}$, Siamak Noroozi ${ }^{5}$ \\ ${ }^{1}$ Faculty of Mechanical Engineering, University of Malaysia Pahang \\ 26600 Pekan, Pahang, Malaysia \\ ${ }^{2}$ Department of Mechanical Engineering, Faculty of Engineering \\ University of Malaya, 50603 Kuala Lumpur, Malaysia \\ *Email: mikeson.khoo@yahoo.com; khooshinyee@um.edu.my \\ Phone: +60379675270; Fax: +60379675317 \\ ${ }^{3}$ Department of Civil Engineering, Faculty of Engineering \\ University of Malaya, 50603 Kuala Lumpur, Malaysia \\ ${ }^{4}$ Advanced Shock and Vibration Research Group, \\ Applied Vibration Laboratory, Block R, Faculty of Engineering \\ University of Malaya, Malaysia \\ ${ }^{5}$ School of Design, Engineering \& Computing, Bournemouth University \\ Poole, Dorset, BH12 5BB, UK
}

\begin{abstract}
Previous impact force identification has focused on collocated cases because noncollocated cases tend to be ill-posed. Considering the impact location is inaccessible, impact force identification using remote responses away from the impact location must be developed. This study initiates an effort to examine impact force identification for non-collocated case. A methodology utilizing operating deflection shape analysis, modal analysis and the modal transformation method (MTM) is presented to identify the unknown dynamic force. The performance of this approach is examined via experimental verification. The objective of this study is to examine the effectiveness of impact force identification by using MTM for both collocated and non-collocated cases. By measuring the response and frequency response function of the test rig, the time history of the unknown force is recovered by the force identification method where the impact location is known. The proposed method is examined at Points 1 and 15, which have satisfactory and poor curve fitting results respectively. It is found that force accuracy improves when the curve fitting result is enhanced. Experimental results show that impact force identification via MTM is applicable in both collocated and noncollocated cases, only if the curve fitting results satisfactory.
\end{abstract}

Keywords: Frequency response function; impact force identification; modal analysis; modal transformation method; operating deflection shape analysis.

\section{INTRODUCTION}

Impact force is the main cause of material fatigue of many structures, and particularly in lightweight structures (Liu \& Han, 2004). Impact force identification has therefore become one of the key issues in structural design (Adekunle, Adebiyi, \& Durowoju, 2013; Khoo et al., 2014). Force identification using the inverse method is important 
when direct measurement using force sensors is not possible due to difficulties of installation and dynamic characteristic altering problems (Yoon \& Singh, 2011). The analysis involved backtracking to determine the unknown force can be done based on responses measured at a series of locations and the dynamic characteristics of a system. These two important parameters can be obtained by using operating deflection shape (ODS) analysis and modal analysis (MA). This analysis is known as impact force identification with modal transformation method (MTM) (Jamil, Yusoff, \& Mansor, 2012; Rahman et al., 2014; Sani et al., 2010; Sani, Rahman, Noor, Kadirgama, \& Izham, 2011). In fact, the solution of impact force identification may be ill-posed (i.e. the solution is sensitive to measurement noise and hence it is unstable (Chen \& Yuan, 2010). In other words, an ill-posed problem is the case where a small variation in response will result in large changes of identified force and cause meaningless force identification results. An ill-posed problem can occur when there is a lack of information (i.e. when a problem of load identification is not collocated (Uhl, 2007). Uhl (2007) reported that a non-collocated problem would occur if at least one of the loads did not have a distinguishable influence on any of the sensors (i.e. the remote sensors are far away from the impact location and none are located at the impact).

Previous impact force identification Hundhausen, Adams, and Derriso (2007); (Rahman et al., 2014) was mainly applied to collocated cases. This study initiates an effort to examine impact force identification for non-collocated case. Considering the impact locations are inaccessible (i.e. bump-excited impact force on a vehicle), a noncollocated force identification method had to be performed by using responses collected at remote points. In this paper, impact force identification using MTM is demonstrated to identify force from remote accelerometers via experimental verification. The impact location is known. The efficiency of this approach is compared to the results in a collocated case. The objective of this study is to examine the effectiveness of impact force identification using MTM in both collocated and non-collocated cases.

\section{MATERIALS AND METHODS}

\section{Set-up of Experiment Equipment}

A rectangular Perspex test rig with four ground supports is referred to as the automobile test rig. Fifteen accelerometers are attached tothe rig and numbering is as shown in Figure 1. Theseare used to measure responses due to impact force. Multiple sensorsare used to prevent roving mass loading effect. A modally tuned impact hammer is used to acquire the impact excitation signal. The input and output signals are connected to a laptop through a data acquisition (DAQ) system. Post-processing of the raw data was performed using DASYLab ${ }^{\circledR}$ and MATLAB ${ }^{\circledR}$ software.

\section{Impact Force Determination By Using Modal Transformation Method}

Modal analysis is a technique to determine the inherent dynamic characteristics of a structure, which are comprehensively defined by natural frequencies, mode shapes and damping (Halvorsen \& Brown, 1977). Once the modal parameters are obtained through the curve fitting of a single column raw frequency response function (FRF) matrix, MTM is applied to synthesize the FRF as shown in Eq. (1). Given the number of responses, modes and force measurements are $n, m$ and $f z$ respectively: 


$$
\underset{n x f z}{\mathbf{G}(\omega)]}=\left[\underset{n \times m}{\left[\mathbf{\Phi}_{\mathbf{N}}\right]}\left[\because 1 /\left(-\omega^{2}+\underset{m x m}{2 i \omega \zeta_{k}} \omega_{0, k}+\omega_{0, k}^{2}\right) \cdot\right] \underset{m x f z}{\left[\mathbf{\Phi}_{\mathbf{N}}\right.}\right]^{\mathrm{T}}(1)
$$

where $[\mathbf{G}(\omega)]$ is an $n$ by $f z$ synthesized FRF matrix. $\left[\boldsymbol{\Phi}_{\mathbf{N}}\right]$ is $n$ by $m$ unit modal mass (Gaskell, Summers, Thompson, \& Savage) mode shape matrix due to response DOF. It can be obtained from residue mode shape, following Richardson (2000). $\left[\mathbf{\Phi}_{\mathbf{N}}\right]^{\mathrm{T}}$ ism by $f z$ UMM mode shape matrix due to force DOF and it is transposedas $\left[\boldsymbol{\Phi}_{\mathbf{N}}\right] . \omega_{0, k}$ is the $k^{\text {th }}$ mode natural frequency where $k=1,2, \ldots, m . \zeta_{k}$ is the $k^{\text {th }}$ mode damping ratio. $[\because \bullet$ ] is a diagonal matrix. $\omega$ is angular frequency.

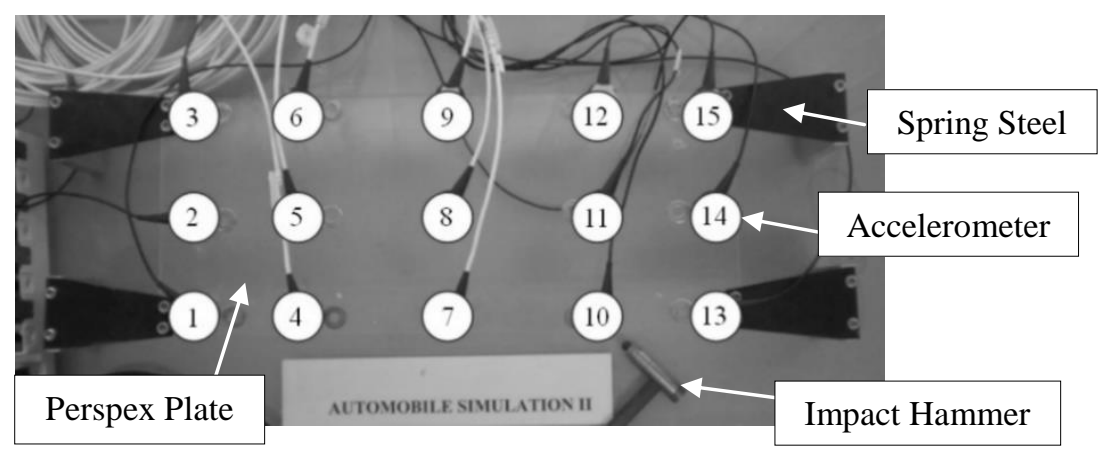

Figure 1.Point numbering on the test rig.

Unknown force can be recovered by multiplying pseudo-inverse, pinv of synthesized FRF matrix to the measured response vector using Eqs. (2) and (3). To obtain a least square solution of force identification, it must satisfy $n \geq m \geq f z$.

$$
\begin{aligned}
& \underset{f \times x 1}{\boldsymbol{Q}(\omega)}\}=\operatorname{pinv}\{[\underset{n x f z}{\mathbf{G}(\omega)]}\{\underset{n \times 1}{\ddot{\boldsymbol{X}}(\omega)}\} \\
& \operatorname{pinv}\{[\mathbf{G}(\omega)]\}=\operatorname{inv}\left\{[\mathbf{G}(\omega)]^{\mathrm{h}}[\mathbf{G}(\omega)]\right\}[\mathbf{G}(\omega)]^{\mathrm{h}}
\end{aligned}
$$

where $\{\ddot{\boldsymbol{X}}(\omega)\}$ and $\{\boldsymbol{Q}(\omega)\}$ are $n$ by 1 acceleration vector and $f$ by 1 force vector respectively. $\omega$ is angular frequency. The inv is the direct inverse method. $\cdot^{\mathrm{h}}$ is the complex conjugate transpose of a matrix.

The force identification for collocated cases is shown in Eq. (4). Using MTM, non-collocated (i.e. force and response locations are different) responses are sufficient for estimating the force. This means that force determination can be made by using remote responses that are far from the impact location. This is illustrated in Eq. (5). Note that force at Point 1 can be calculated from responses other than Point 1.

$$
\left\{\begin{array}{c}
Q_{1} \\
Q_{2} \\
\vdots \\
Q_{n}
\end{array}\right\}=\operatorname{pinv}\left[\begin{array}{cccc}
G_{1: 1} & G_{1: 2} & \cdots & G_{1: f z} \\
G_{2: 1} & G_{2: 2} & \cdots & G_{2: f z} \\
\vdots & \vdots & \ddots & \vdots \\
G_{n: 1} & G_{n: 2} & \cdots & G_{n: f z}
\end{array}\right]\left\{\begin{array}{c}
\ddot{X}_{1} \\
\ddot{X}_{2} \\
\vdots \\
\ddot{X}_{n}
\end{array}\right\}
$$




$$
\left\{\begin{array}{c}
Q_{1} \\
Q_{2} \\
\vdots \\
Q_{n}
\end{array}\right\}=\operatorname{pinv}\left[\begin{array}{cccc}
G_{2: 1} & G_{2: 2} & \cdots & G_{2: f z} \\
G_{3: 1} & G_{3: 2} & \cdots & G_{3: f z} \\
\vdots & \vdots & \ddots & \vdots \\
G_{n: 1} & G_{n: 2} & \cdots & G_{n: f z}
\end{array}\right]\left\{\begin{array}{c}
\ddot{X}_{2} \\
\ddot{X}_{3} \\
\vdots \\
\ddot{X}_{n}
\end{array}\right\}
$$

According to (Rahman et al., 2014), the force identification problem becomes well-posed once impact location is known a priori (i.e. impact location at Point 1). Thus Eq. (5) is reduced to Eq. (6) if the impact force is acting at Point 1.

$$
\left\{Q_{1}\right\}=\operatorname{pinv}\left\{\begin{array}{c}
G_{2: 1} \\
G_{3: 1} \\
\vdots \\
G_{n: 1}
\end{array}\right\}\left\{\begin{array}{c}
\ddot{X}_{2} \\
\ddot{X}_{3} \\
\vdots \\
\ddot{X}_{n}
\end{array}\right\}
$$

In this study, the force identification method is tested for two cases: collocated and non-collocated. In both cases, the modal parameters are computed from MA where a reference force sensor is acting at an anti-node (i.e. Point 1) and 15 response sensors were located at 15 discrete locations as per Halvorsen \& Brown (1977). Note that the discrete locations must be sufficient to describe the mode shape in the frequency of interest. In the collocated case, a single unknown impact force acting at Point 1 on the test rig is identified from 15 acceleration sensors including the one at impact location. In the non-collocated case, only 14 remote accelerometers are used, excluding the one at impact location. The measured force and identified force are compared to evaluate the accuracy of force identification by using the MTM method in collocated and noncollocated cases. This procedure is repeated to identify the unknown impact force acting at Point 15. Note that impact locations at Point 1 and Point 15 have different curve fitting results. The correlations between the imaginary part of the measured FRFs and synthesized FRFs for force DOF 1 and 15 are calculated as 0.28 and 0.53 respectively, as shown in (Rahman et al., 2014). In this study, the former case is considered a poor curve fitting result, while the latter case is considered as satisfactory curve fitting result. It is worth noting that due to the limitations of the curve fitting algorithm (Vibrant Technology Inc., 2012), it is difficult to obtain correlation in the range of $0.9-1.0$.

\section{RESULTS AND DISCUSSION}

Two sets of impact force identification results at different impact locations (Point 1 and Point 15) have been obtained for both collocated and non-collocated cases. The identified forces for the collocated and non-collocated cases are compared to the measured force. The force identification results for impact locations at Point 1 and Point 15 are shown in Figures 2 and 3 respectively. From Figure 2, it is seen that the identified force for non-collocated case has a larger jump and oscillating component compared to collocated cases. The percentage of errors between the amplitude of identified force and measured force are $69.93 \%$ and $49.00 \%$ respectively. The impact function of recovery force is satisfied for both cases, however the accuracy of magnitude is not satisfied. This is primarily due to the poor curve fitting result for impact location at Point 1.

From Figure 3, it is observed that the identified force for non-collocated case has a lower amplitude compare to collocated case. The percentage of errors between 
identified force and measured force are $-9.12 \%$ and $-14.40 \%$ respectively. The impact function of identified force is correct for both cases. The force identification result at Point 15 is satisfactory for both cases because it has a satisfactory curve fitting result.

Combining results from Figures 2 and 3, it is found that the accuracy of force identification dropped in term of amplitude when non-collocated case is used. Besides, it is found that the accuracy of force identification result via MTM can be varied for different impact locations. Result shows that the variation between the accuracy of force identification at Point 15 for both collocated and non-collocated cases is smaller compared to the force identification result at Point 1 . The variations are $20.93 \%$ and $5.28 \%$ for force identification result at Point 1 and Point 15 respectively. This shows that the force identification at Point 15 is much more accurate than force identification at Point 1.

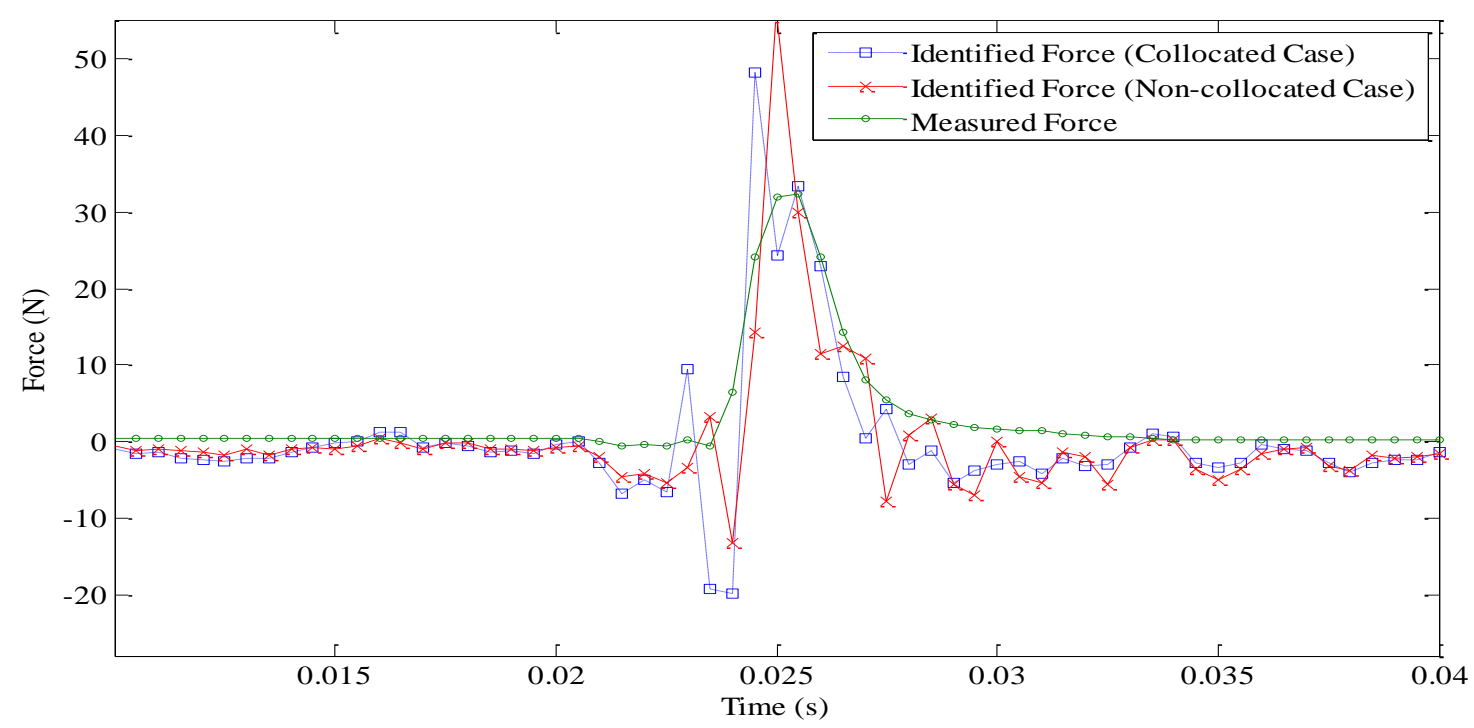

Figure 2. Comparison between identified force and measured force at Point 1 for collocated and non-collocated cases.

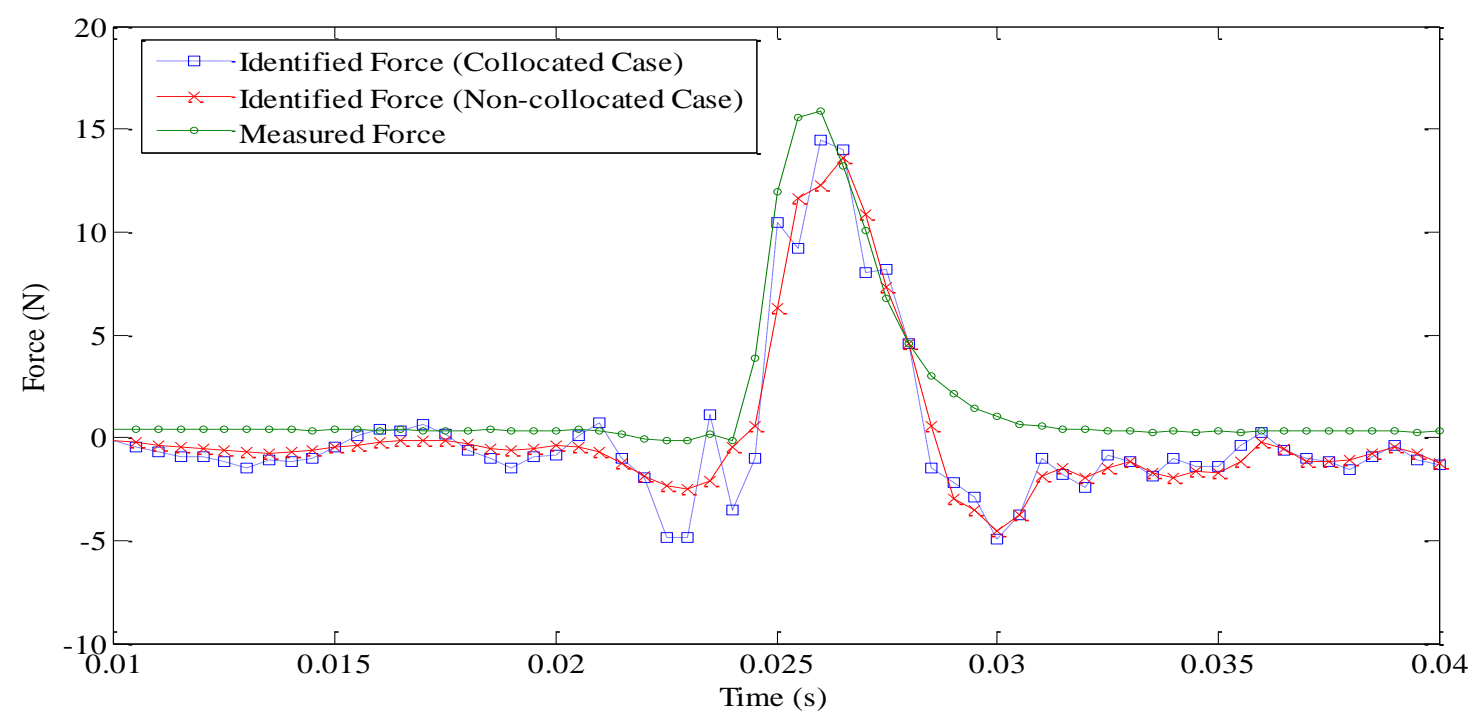

Figure 3.Comparison between identified force and measured force at Point 15 for collocated and non-collocated cases. 
The correlation coefficients for collocated and non-collocated cases due to impact locations at Points 1 and 15 are shown in Table 1. Note that the correlation coefficients are calculated based on the measured force and identified force in time domain. In the case of impact force identification, only a correlation coefficient greater than 0.90 is described as good in terms of force accuracy, correlation coefficient greater than 0.85 is described as satisfactory result. From Table 1, result shows that correlation coefficients for force identification at Point 1is less than 0.85 for both collocated and non-collocated cases. Thus, the force identification result is not acceptable. This shows that the poor curve fitting result affects the force accuracy at Point 1. Besides, the correlation coefficients for force identification at Point 15 is more than 0.85 for both collocated and non-collocated cases. Thus, the force identification result is satisfactory. This shows that a satisfactory curve fitting result will eventually produce a satisfactory force identification result at Point 15. By comparing the force identification results at Points 1 and 15, it was found that force accuracy in terms of the correlation coefficient improves when the curve fitting result is enhanced. This explains why the force identification result at Point 15 is better than at Point 1, as shown in Table 1.Furthermore, the accuracy of force identification for non-collocated case is slightly greater than for the non-collocated cases, at all examined impact locations in terms of correlation coefficient. This shows that impact force identification using MTM is applicable in both cases, only if the curve fitting result can be enhanced to a satisfactory level. In future, a higher correlation of curve fitting result must be obtained to further improve the impact force identification result to a good level.

Table 1.Correlation coefficients for collocated and non-collocated cases according to impact locations 1 and 15.

\begin{tabular}{lcccc}
\hline Impact Location & \multicolumn{3}{c}{15} \\
\hline Type of Case & Collocated & Non-Collocated & Collocated & Non-Collocated \\
\hline Correlation Coefficients & 0.77 & 0.80 & 0.85 & 0.87 \\
\hline
\end{tabular}

\section{CONCLUSIONS}

The effectiveness of impact force identification using MTM has been examined in both collocated and non-collocated cases, where it is satisfactory for both cases, only if a satisfactory curve fitting result is used. The proposed method has been examined at two discrete impact locations (Points 1 and 15), which have poor and satisfactory curve fitting results respectively. Impact force identification via MTM fails if a poor curve fitting result is used, where the correlation coefficients for both collocated and noncollocated cases are less than 0.85 . Further research will be conducted to overcome the limitations of the current curve fitting algorithm and enhance the accuracy of force identification results to a good level (i.e. correlation coefficients greater than 0.9).

\section{ACKNOWLEDGEMENTS}

The authors wish to acknowledge the financial support and advice given by the Postgraduate Research Fund (PV086-2011A), Advanced Shock and Vibration Research (ASVR) Group of the University of Malaya. 


\section{REFERENCES}

Adekunle, A. S., Adebiyi, K. A., \& Durowoju, M. O. (2013). Impact of quench severity and hardness on aisi 4137 using eco-friendly quenchants as industrial heat treatment. Journal of Mechanical Engineering and Sciences, 4, 409-417.

Chen, C., \& Yuan, F.-G. (2010). Impact source identification in finite isotropic plates using a time-reversal method: Theoretical study. Smart Materials and Structures, 19(10), 105028.

Gaskell, P., Summers, J., Thompson, H., \& Savage, M. (1996). Creeping flow analyses of free surface cavity flows. Theoretical and Computational Fluid Dynamics, $8(6), 415-433$.

Halvorsen, W. G., \& Brown, D. L. (1977). Impulse technique for structural frequency response testing. Sound and Vibration, 11(11), 8-21.

Hundhausen, R. J., Adams, D. E., \& Derriso, M. (2007). Impact loads identification in standoff metallic thermal protection system panels. Journal of intelligent material systems and structures, 18(6), 531-541.

Jamil, N., Yusoff, A. R., \& Mansor, M. H. (2012). Literature review of electromagnetic actuator force generation for dynamic modal testing applications. Journal of Mechanical Engineering and Sciences, 3, 311-319.

Khoo, S., Ismail, Z., Kong, K., Ong, Z., Noroozi, S., Chong, W., \& Rahman, A. (2014). Impact force identification with pseudo-inverse method on a lightweight structure for under-determined, even-determined and over-determined cases. International Journal of Impact Engineering, 63, 52-62.

Liu, G.-R., \& Han, X. (2004). Computational inverse techniques in nondestructive evaluation. Florida: CRC press.

Rahman, A., Ghaffar, A., Yee, K. S., Ismail, Z., Tong, C. W., \& Noroozi, S. (2014). Impact force identification by using modal transformation method for automobile test rig. Applied Mechanics and Materials, 471, 102-106.

Richardson, M. H. (2000). Modal mass, stiffness and damping. Vibrant Technology, Inc., Jamestown, CA, 1-5.

Sani, M. S. M., Noor, M. M., Zainury, M. S. M., Rejab, M. R. M., Kadirgama, K., \& Rahman, M. M. (2010). Investigation on modal transient response analysis of engine crankshaft structure WIT Transactions on the Built Environment (Vol. 112, pp. 419-428).

Sani, M. S. M., Rahman, M. M., Noor, M. M., Kadirgama, K., \& Izham, M. H. N. (2011). Identification of dynamics modal parameter for car chassis IOP Conference Series: Materials Science and Engineering (Vol. 17, pp. 1-8).

Uhl, T. (2007). The inverse identification problem and its technical application. Archive of Applied Mechanics, 77(5), 325-337.

Vibrant Technology Inc. (2012). Fourier spectra, auto spectra, and psds; curve fitting; mode indicator methods; curve fitting methods; curve fitting guidelines; counting the modes in a band. Estimating frequency \& damping; estimating residues; saving shapes. Product Help, ME'Scope V4.0.0.99.

Yoon, J.-Y., \& Singh, R. (2011). Estimation of interfacial forces in a multi-degree of freedom isolation system using a dynamic load sensing mount and quasi-linear models. Journal of Sound and Vibration, 330(18), 4429-4446. 